\title{
APROXIMACIÓN TEÓRICA A LA RELACIÓN ENTRE DESARROLLO HUMANO Y RACIONALIDAD ECONÓMICA (Theoretical approach to the relationship between human development and economic rationality)
}

\author{
Fecha de recepción: 20 octubre de 2015 \\ Fecha de aceptación: 16 febrero de 2016 \\ Encuentre este artículo en \\ http://biblioteca.uniminuto.edu/ojs/index.php/IYD/issue/archive \\ Para citar este artículo / To cite this article. \\ Vera-Ramírez, H. (2016). Aproximación teórica a la relación entre \\ desarrollo humano y racionalidad económica. Revista Inclusión \& \\ Desarrollo, 3 (I), 67-79
}

Henry Daniel Vera Ramírez ${ }^{\prime}$

hdverar@unicolmayor.edu.co

Resumen

La racionalidad económica, en el marco general de las teorías que se enfocan en el tema, puede entenderse generalmente como un método de elección entre alternativas o, lo que es lo mismo, como un sistema de gestión de preferencias entre los diferentes objetos económicos; en este sentido, entonces, implica la necesaria conexión entre la economía y la psicología de la conducta, lo que abre nuevas posibilidades de análisis mediante un diálogo interdisciplinar que permite comprender la fuente de motivación de los fenómenos económicos, los métodos o las formas de elección, y los diferentes acuerdos que los individuos de forma heterogénea establecen. De igual manera, los problemas de elección se relacionan con la capacidad que tiene el individuo para tomar decisiones, que siempre será mayor en la medida en que posea un bagaje más amplio de conocimientos que la potencie. El presente artículo pretende reflexionar sobre los puntos de encuentro entre la racionalidad económica y la forma en la cual el paradigma del desarrollo humano - desde el enfoque de las capacidades- provee un campo de acción y permite una elección de alternativas que generan al individuo mayor bienestar, más allá del paradigma utilitario. Para el estudio del concepto de desarrollo humano y el enfoque de capacidades, se tomarán como autores básicos a Sen (1996, 1997 y 1999), Nussbaum (1996) y Max Neef (1993a y 1993b); en cuanto al estudio de la racionalidad económica, se hará énfasis en los planteamientos de Kahneman y Tversky (1974, 1979 y 1981).

Palabras clave: desarrollo, racionalidad, utilitarismo, elección.

Abstract

Economic rationality, within the general framework of the theories that focus on the subject, can generally be understood as a method of choice among alternatives, or what is the same, as a management system of preferences among different economic objects. Economic rationality then implies, the necessary connection between economics and psychology of behavior, which opens new possibilities for analysis by means of an interdisciplinary dialogue that allows us to understand the source of motivation of economic phenomena, methods or forms of choice and the various agreements that individuals establish heterogeneously. Similarly, election problems have to do with the ability of the individual to make decisions that will always be better to the extent that has a greater wealth of knowledge that enhances his/her capabilities. This article aims to reflect on the points of contact between economic rationality and the way in which the human development paradigm, -from capabilities approach - provides a field of action and allows a choice of alternatives that generate a greater individual well-being, beyond the utilitarian paradigm. For the study of the concept of human development and capability approach Sen (1996, 1997 and 1999), Nussbaum (1996) and Max Neef (1993a and 1993b) will be the authors of reference. For the study of economic rationality, the approaches of Kahneman and Tversky (1974, 1979 and 198I) will be the emphasized.

Keywords: development, rationality, utilitarianism, choice.

'Magister en Investigación Social Interdisciplinaria de la Universidad Distrital, Psicólogo de la Universidad Nacional de Colombia y Economista de la Universidad Militar Nueva Granada. Docente investigador de la Universidad Colegio Mayo de Cundinamarca. 


\section{INTRODUCCIÓN}

EI abordaje de un tema como el desarrollo - humano implica una gran revisión de conceptos y planteamientos teóricos que engloban, a su vez, nuevas corrientes teóricas y discusiones centradas en la búsqueda de la redefinición del concepto mismo de desarrollo. Diferentes tendencias acerca de esta noción han enfatizado en la medición de variables de tipo cuantitativo, bajo el supuesto de que los agentes económicos actúan de manera racional.

Después de la tendencia inicial, acaecida en la primera mitad del siglo $X X$, que consistía en observar el desarrollo de los países como un resultado -algunas veces espurio-, de estos análisis cuantitativos acerca de diferentes indicadores económicos y que se constituían como fuente única y universal para definir el estado de desarrollo de diferentes regiones o países, los nuevos estudios sobre desarrollo humano han aportado a esta perspectiva no solo la valoración de los recursos económicos con los que cuenta un determinado país -medidos generalmente por el producto nacional-, sino el aporte que realizan otras tendencias que hacen énfasis en la educación y en la formación de capital humano, como por ejemplo el enfoque de capacidades, introduciendo la revalorización de nuevos conceptos como el de conocimiento y la ubicación de este como recurso de gran valor para el avance de las diferentes regiones, países, naciones o comunidades. Sin embargo, estas nuevas tendencias no niegan la importancia de definir la distribución de recursos de forma racional, de tal manera que las decisiones de política redistributiva, por ejemplo, deben hacerse con criterios técnicos, epistemológicamente fuertes y en armonía con las nuevas tendencias. La una no excluye a la otra; de allí la importancia de estudiar el concepto de racionalidad económica.

Por otro lado, la adopción por parte del PNUD² de esta perspectiva en la realización de sus informes sobre desarrollo humano, ha generado la revalorización de la importancia del vínculo entre el concepto de desarrollo humano y las elecciones del homo œconomicus. Esta racionalidad económica, en el marco de teorías que la abordan, puede entenderse generalmente como un método de elección entre diferentes alternativas o un sistema de ordenación de preferencias entre diferentes objetos económicos, lo que implica la necesaria conexión entre la ciencia económica y la psicología del comportamiento, abriendo posibilidades de análisis, mediante un extenso diálogo de saberes, para la comprensión del origen motivacional de algunos fenómenos económicos, métodos o formas de elección y de las diferentes ordenaciones que el individuo establece sobre las heterogéneas opciones que se le presentan.

\section{PROBLEMA}

En este texto se considerará como punto de partida una idea importante: las preferencias se refieren a objetos económicos -se puede decir necesidades y recursos-, y la forma en la que el individuo decide su ordenación se basa generalmente en estimaciones sobre su valor y coste. El problema radica en la forma o la metodología que se implemente para la medición de esos costos y valores. La intención, en este momento, no es generar una discusión acerca de una definición muy amplia o la de adentrarse en una teoría del valor, ya que cada sistema económico tiene asociada una forma distinta de estimar valores y costes, de lo que se podría inferir que necesariamente cada sistema económico asocia a sí mismo una racionalidad diferente. Un conjunto de lo que se ha definido como objetos económicos puede presentar diferentes ordenaciones bajo diferentes sistemas económicos, razón por la cual podría afirmarse que la racionalidad económica dependerá de la valoración de estos objetos económicos y de las ordenaciones que haga cada individuo según al grupo social al que pertenezca. Dicha valoración no escapa, sin embargo, a las consideraciones subjetivas del decisor o agente económico que actúa -racionalmente o no-. Estos elementos de índole subjetiva generan un nuevo espacio de discusión en torno a la elección entre objetos económicos que van desde la neuroeconomía hasta la teoría de la decisión, los cuales son campos que están más allá de las posibilidades del presente artículo.

Sobre la base de estas premisas fundamentales, se propone, en principio, analizar el contexto histórico en el que se ha desarrollado el paradigma del desarrollo humano, para contextualizar su avance teórico, y las propuestas de Sen (1997 y 1999) y Nussbaum (1996). La revisión hermenéutico-interpretativa de los autores mencionados permitirá encontrar los puntos de conexión entre las diferentes posturas y planteamientos, que en principio parecerían no tener ninguna relación.

2Programa de las Naciones Unidas para el Desarrollo. 


\section{PerspeCtiva HISTÓRICA ACERCA DEL \\ CONCEPTO DE DESARROLLO HUMANO}

Intentar adentrarse en el concepto de desarrollo humano, implica reconocer la existencia de un conjunto de corrientes variopintas que tratan de devolver a la ciencia fría su carácter humano; no obstante, en este artículo no se problematizará acerca de la influencia que cada enfoque existente ha llegado a tener. Sin embargo, al analizar el proceso histórico que deriva en la adopción de la perspectiva del desarrollo humano por parte del PNUD, se deben observar algunos periodos por los cuales han transitado las diferentes nociones acerca del concepto de desarrollo. Para Sen (1999), si se analizara de manera general el proceso de evolución de las diferentes corrientes teóricas que han abordado el tema, podríamos encontrar como básicas las siguientes etapas:

- En una primera etapa, a finales del siglo XVIII, la atención del desarrollo se concentraba en el individuo, sobre todo a partir de la llamada "escuela de la economía clásica y liberal", también conocida como "escuela de Manchester", cuya premisa básica consistía en que el egoísmo y el individualismo del ser humano tendrían como consecuencia la búsqueda de la riqueza individual, lo que generaría, por lo tanto, la riqueza colectiva.

- Luego, en una segunda etapa, que llegaría hasta la primera mitad del siglo XX, el desarrollo se concentraría en lo eminentemente económico, en la producción de riqueza y en la medición de la misma, con enfoques básicamente cuantitativos, sin la consideración de otros aspectos como la educación, la salud, el bienestar social, etc.

- En una última fase, a partir de la década de los setenta, los estudios se concentrarían en el desarrollo socioeconómico, con un nuevo énfasis en la pobreza, indagando acerca de su origen y orientándose hacia el desarrollo humano. En esta última etapa se haría énfasis en el desarrollo de oportunidades y se volvería a centrar la atención en la importancia de los individuos; pero, a diferencia de los enfoques clásicos, no en el individuo aislado sino como parte de la comunidad y miembro de una red social que debería garantizar su libertad.

Estas etapas han reflejado cambios en la medición del desarrollo ya que no se han anclado en la definición del mismo como la expansión de los productos primarios y de la riqueza, sino como la ampliación de las opciones del hombre para garantizar su libertad. Si se sigue esta perspectiva histórica se observa, como piedra angular, la revaloración del individuo como objetivo del desarrollo, que había sido menos evidente sobretodo en la segunda fase. Como anota Hernández (2004), la nueva perspectiva del desarrollo humano ha permitido extender las potencialidades del ser humano, facilitándole desplegarse como ser en libertad y no solo en la garantía de sus derechos más básicos e importantes. Es en esta perspectiva histórica que se hace necesaria la definición de desarrollo humano.

El desarrollo humano significa, entonces, que todas las personas deberían tener la posibilidad de desplegar a plenitud sus capacidades vitales, pero no en función de un estándar abstracto y universal, válido sin distingo alguno para todos los grupos humanos, sino de aquello que los sujetos del desarrollo -que siempre deben ser las personas o los individuos- más valoren. Este nuevo giro, que consiste en definir los objetivos del desarrollo con base en lo que las personas más valoran, es uno de los grandes aportes de la categoría de desarrollo humano, por cuanto le confiere un papel privilegiado a las dimensiones subjetivas y remite a la necesidad de valorar las diferencias humanas. En esta nueva perspectiva se encuentra implícito el abandono de la visión unilineal y ascensional del crecimiento económico dominante en las teorías tradicionales del desarrollo signadas por la creencia, eurocéntrica y moderna, de que todas las sociedades debían atravesar más o menos las mismas etapas hasta alcanzar el modelo ideal de desarrollo definido por un modelo -también ideal-, de sociedad y de cultura, constituido principalmente por los países desarrollados de Occidente. Uno de los puntos de la discusión tendría que ver con la consideración de qué es lo que más valoran los individuos en la sociedad y en el establecimiento de ordenaciones de valores que permitiesen un desarrollo colectivo. Por ejemplo, la protección a la infancia y el acceso a la educación, se inscriben como una de las ordenaciones de valores supremos de las sociedades, y la forma en la que se desarrolla dicha protección y dicha garantía podría constituirse en un indicador de desarrollo humano.

Por otro lado, es ya común escuchar que el desarrollo humano está íntimamente relacionado con la calidad de vida. Max Neef (1993), en la primera etapa de su pensamiento, centra su enfoque de desarrollo en las necesidades humanas relacionadas con la calidad de vida, planteando tres postulados:

- El desarrollo se refiere a las personas y no a los objetos.

- El mejor proceso de desarrollo es aquel que permita elevar más la calidad de vida de las personas. 
- La calidad de vida dependerá de las posibilidades que tengan las personas de satisfacer adecuadamente sus necesidades humanas fundamentales.

Esta perspectiva contribuye a los enfoques anteriores y se constituye en un planteamiento inicial para el reconocimiento de la calidad de vida como instrumento para el desarrollo, a partir de las potencialidades del ser humano, sin desconocer sus necesidades básicas. Por su parte, Sen (1999) ha aportado la perspectiva del desarrollo como libertad, una concepción innovadora que se aleja sustancialmente del enfoque de las necesidades básicas insatisfechas. En sus propias palabras, "(...) el desarrollo puede concebirse (...) como un proceso de expansión de las libertades reales de las que disfrutan los individuos" (Sen, 1999. p. 87).

En otras palabras, el desarrollo no debe medirse con otro indicador que no sea el aumento de las libertades de los individuos. En esta línea, y como anota Edo (2002), la contribución de la libertad al desarrollo puede verse de dos maneras distintas: como un fin tanto como un medio. Sen (1999) incluye estos dos aspectos en su definición del desarrollo como libertad, concediéndole mayor importancia a la primera -como fin-.

La libertad es, ante todo, el fin principal del desarrollo; no se pretende, entonces, aumentar la libertad para lograr algo más, sino por la libertad en sí misma, lo que el autor denomina el "papel constitutivo" de la libertad en el desarrollo. En cuanto a la libertad, como medio, sostiene que, además de ser el objetivo último del desarrollo, puede sery de hecho se constituye en una herramienta para lograrlo; se trata de lo que se define como "papel instrumental" de la libertad en el desarrollo, que se refiere a la forma en que contribuyen los diferentes tipos de derechos básicos y oportunidades a expandir la libertad del hombre en general $y$, por lo tanto, a fomentar el desarrollo. En palabras del propio Sen: "La eficacia de la libertad como instrumento reside en el hecho de que los diferentes tipos de libertad están interrelacionados y un tipo de libertad puede contribuir extraordinariamente a aumentar otros" (Sen, 1999, p. 105).

Otro de los puntos importantes es la relación que establece Sen (1997), entre el capital humano y el desarrollo humano. En los análisis económicos modernos se ha dejado de ver al proceso de desarrollo como una acumulación de capital en términos físicos, para comenzar a considerarlo como un proceso en el que interviene íntegramente la capacidad productiva de los seres humanos. Se considera que variables como la educación y el aprendizaje influyen fuertemente en el proceso de desarrollo. Este cambio en la visión del desarrollo se ha denominado "capital humano". En la literatura se ha confundido, a veces, esta nueva perspectiva del desarrollo con la de Sen; sin embargo, el mismo autor sostiene que esta visión es distinta a la propuesta por él. Ambos enfoques parecen hablar de cosas parecidas pero poseen diferencias profundas; la principal es que la literatura sobre capital humano tiende a centrar la atención en la capacidad de las personas de contribuir al aumento de la producción. Por el contrario, la visión del desarrollo como libertad valora la capacidad (libertad fundamental) de los individuos para vivir la vida que tienen a partir de razones para valorar y para aumentar las opciones reales entre las que puedan elegir.

Por lo tanto, la evaluación centra la atención en logros diferentes: aumentar la producción o llevar adelante la vida que tienen razones para valorar. En el fondo, esto supone una cuestión más profunda: el enfoque de desarrollo como libertad pone a los individuos en el centro de la escena, mientras que el enfoque del capital humano pone en ese lugar al crecimiento económico. Según Sen, "(...) debemos ir más allá del concepto del capital humano una vez reconocidos su importancia y su alcance. La ampliación necesaria (de la libertad) es adicional e inclusiva y en modo alguno una alternativa a la perspectiva del capital humano" (Sen, 1999, p. 127).

Pero, el punto de inflexión que no permitió que los estudios teóricos acerca del desarrollo humano terminasen en el vacío epistemológico, sino que se constituyeran en verdaderos cambios conceptuales y teóricos, es la adopción del PNUD de esta nueva corriente de pensamiento que revaluaba las mediciones tradicionales del desarrollo. Como afirma Hernández (2004):

(...) El enfoque sobre Desarrollo Humano adoptado por el PNUD, siguiendo a Amartya Sen, ha significado un profundo cambio conceptual, a partir del cual se pueden articular nuevas propuestas de políticas públicas, siempre expuestas al momento de su evaluación en la práctica gobernante (p. 59).

La adopción por parte del PNUD, sobre todo a partir de la década de los noventa, del concepto de desarrollo humano como punto de partida para el análisis de la situación de diferentes países emergentes se ha considerado un acierto, porque con él se abandona el tradicional esquema de definir la pobreza de un país a partir de indicadores homogéneos y simples como ingresos y producto per cápita.

Para Argibay, Celorio y Celorio (1997), las experiencias de varios países en desarrollo ponían 
claramente de manifiesto que el crecimiento del ingreso no había resuelto de manera eficaz el problema de la pobreza masiva. El concepto de desarrollo, entonces, orienta su preocupación hacia lo socioeconómico, buscando la superación de la brecha de la pobreza por medio de la educación y del conocimiento. Este avance obliga a introducir nuevos indicadores del desarrollo como la mortalidad y la morbilidad, y otros, relacionados con factores sociales, como el urbanismo, o con factores estrictamente económicos. En las dos últimas décadas se introduce un nuevo índice de desarrollo que tiene que ver con la calidad de vida física, diseñado por el PNUD, que busca concentrarse en el desarrollo como un bienestar alcanzado. Se acogen, entonces, tres nuevos indicadores: la mortalidad infantil, la esperanza de vida y el alfabetismo. La adopción de estas nuevas perspectivas ha logrado difundir en los países en desarrollo una visión del desarrollo humano más holística e integradora de las potencialidades del ser humano y de la promoción de la libertad, como fin y medio para el desarrollo. Sin embargo, el desarrollo humano, además de estar acompañado por una mejora sustantiva en la calidad de vida, debe potenciar las capacidades y aumentar los niveles de libertad.

\section{DESARROLLO HUMANO Y CALIDAD DE VIDA:}

\section{UN ENFOQUE CONTRACTUALISTA}

La concepción de desarrollo humano planteada por Sen (1996, 1997 y 1999) implica una mejora sustantiva en la calidad de vida. En tanto, la concepción de calidad de vida que plantea Nussbaum (1996) también resulta enriquecedora, pues para la autora existen múltiples libertades; por ejemplo, la que permite conducir las relaciones personales y sociales, o la que permite conducir las relaciones de género. Para entender este concepto de libertad es necesario comprender una noción previa que es la de capacidad. El concepto de capacidades básicas tiene como finalidad diferenciar la habilidad para satisfacer ciertos funcionamientos importantes, aun sin ignorar la relevancia de los ingresos, para que se garantice su desarrollo hasta ciertos niveles adecuadamente mínimos. Al respecto, Nussbaum (1996) plantea:

(...) La conversión del ingreso en capacidades básicas puede variar mucho entre los individuos y también entre distintas sociedades, de modo que la posibilidad de alcanzar niveles mínimamente aceptables de las capacidades básicas puede estar asociada con diferentes niveles de ingresos mínimamente adecuados (p. 68).
Lo que resulta difícil es determinar lo que se indica realmente con niveles mínimos, y el impacto de la política pública en la definición de lo que es el nivel mínimo de calidad de vida, de ingresos y de capacidades. De esta manera, para Nussbaum, la habilidad de una persona para lograr varios funcionamientos valiosos puede ser reforzada por las acciones y la política pública; el hecho de poder elegir tiene una importancia para la calidad de vida y el bienestar de una persona (Nussbaum, 1996).

Entre las distinciones por considerar se encuentran, entre otras, el bienestar y la agencia subjetiva, así como el logro y la libertad. Estas categorías de comparación interpersonal e intrapersonal se encuentran interrelacionadas pero no corresponden a lo mismo; el enfoque sobre la capacidad puede usarse para cada uno de estos elementos, pero no con el mismo alcance. La importancia de la perspectiva de las capacidades reside en que esta funciona para la evaluación de las oportunidades y los éxitos individuales, lo que es significativo dentro del pluralismo y el estándar de vida, ya que una teoría pluralista debe considerar como un bien social la posibilidad de que puedan existir múltiples puntos de vista del bien individual, lo que es imposible de incluir en una teoría total o absoluta.

Nussbaum sigue la posición contractualista de John Rawls (2006), considerando que el contrato social se asocia a la utilidad, la buena vida, el respeto a sí mismo, la dignidad, la obligación y el derecho del ciudadano, que se hacen evidentes en el mundo real de los contratos hechos entre personas, y que, a su vez, dependen de ciertas circunstancias y son públicamente comprobables. En esta línea, un contrato no puede hacerse para entregar algo imposible ni para dar algo libremente sin esperar una retribución. Este contrato dependerá del grado de desigualdad según la época y la sociedad, igual que de la clase de servicios que se incluyen en él. Se considera importante incorporar también un aspecto relacionado con la negociación en la que los individuos o las sociedades se pongan de acuerdo. En este sentido:

(...) Los contratos sociales deben serlo para el intercambio de estos productos básicos o servicios: que dependen de circunstancias públicamente comprobables. Y el estándar de vida de los ciudadanos está representado por el dominio que ellos tienen sobre estos productos o servicios primarios, dadas las circunstancias. (...) Se puede decir que el estándar de vida de los individuos consiste en esos componentes de su bienestar, cuyo fortalecimiento sería sujeto apropiado de un 
contrato social entre los individuos que desean compartir los beneficios de la cooperación social (Nussbaum, 1996, p. 513).

Esta visión no es reduccionista, en el sentido de ver el intercambio de productos y servicios como una forma del contrato social, ya que lo que busca realmente es que el intercambio generé un mayor bienestar y una potenciación de las capacidades de los individuos.

Las ideas sobre lo que es una buena vida depende, por ejemplo, del lugar que se otorgue a los productos más importantes (primarios) y a los servicios necesarios para lograrla; de esta manera, un contrato social debe apoyarse en el acuerdo de todas las partes. De igual manera, las consideraciones que se hagan sobre la justicia afectan a las relaciones, por lo que se debe establecer un contrato social diferente que genere una concepción ampliada del estándar de vida.

Hay que considerar, además, que el estándar de vida debe ser sensible a los valores de cada cultura, ya que los individuos están en libertad de ordenar sus preferencias de acuerdo con su propia escala de valores. Según Rawls (2006), la equidad se debe entender como la necesidad de adaptar la justicia a las necesidades propias de cada comunidad humana, ya que estas poseen una fuerte cohesión y presentan un propósito común.

Una correcta política gubernamental, entonces, debe interesarse en el estándar de vida de los ciudadanos. El estándar de vida es el aspecto relacionado con el bienestar de los individuos que se transforma en un interés de la sociedad. Para Nussbaum:

La sociedad es una institución de cooperación entre los individuos para su mutuo beneficio, la sociedad hace posible la implementación de un marco que incluye, por ejemplo, los derechos de propiedad y la organización tanto de la producción como del consumo, sin el que los individuos no podrán gozar de muchas de las actividades que son centrales para su bienestar (Nussbaum, 1996, p. 510).

Con respecto a la valoración de los derechos, se considera que entre más general sea el derecho, más elevada será su defensa y mucho más alta su estimación en la sociedad. En este sentido, el derecho a la propiedad resulta básico para la sociedad, pero no debe restarle importancia al hecho de que la prosperidad de los ciudadanos individuales y su contribución al bienestar colectivo, son cosas a las que la sociedad no puede ponerles valor. Un ejemplo de un elemento axiológico al que no se le puede poner valor es la libertad, pues, según Sen (1999), si bien es un fin último de desarrollo, no es posible medir en ningún tipo de escala lo que ella significa para la mayoría de los grupos sociales en occidente. En la vía de mejorar la calidad de vida, como condición para alcanzar el bienestar colectivo, se puede tener en cuenta la propuesta de olivares (2014) que incluye los elementos que se presentan en la tabla I.

La calidad de vida debe entenderse, entonces, en el marco de tres categorías básicas: el tener, el amar y el ser. La primera categoría tiene que ver con las

\section{Tener}

Tiene que ver con el dominio y el control de las condiciones materiales indispensables para la supervivencia y para no incurrir en la pobreza. Se refiere a las necesidades de nutrición, de ambiente saludable (aire, agua, clima) y de atención de enfermedades, entre otras. Se consideran los siguientes indicadores: los recursos económicos (o ingresos), las condiciones de la vivienda, el empleo, las condiciones y el ambiente de trabajo, la salud y la educación.

\section{Amar}

Se refiere al vínculo de la persona con otras y su identificación como una comunidad. Se utilizan indicadores como arraigo y contacto con la comunidad local, apego a la familia y a los parientes, patrones vigentes de amistad, vínculos con compañeros de asociaciones y organizaciones, y relaciones con compañeros de trabajo.

Tabla I. Tipología de capacidades

Nota: Adaptado de Desarrollo sustentable: Unidad IV, Calidad de Vida y Desarrollo Sustentable, Olivares, 2014.

\section{Ser}

Se trata de la integración social y ambiental, en general, que permite el desarrollo personal y evita el aislamiento. Se incluyen los siguientes indicadores: la participación en las decisiones y las actividades que influyen en su vida, la actividad política, la oportunidad para realizar actividades recreativas, la oportunidad de una vida significativa en el trabajo, de integración y de disfrute de la naturaleza (contemplación, paseos, jardinería, crianza, etc.).

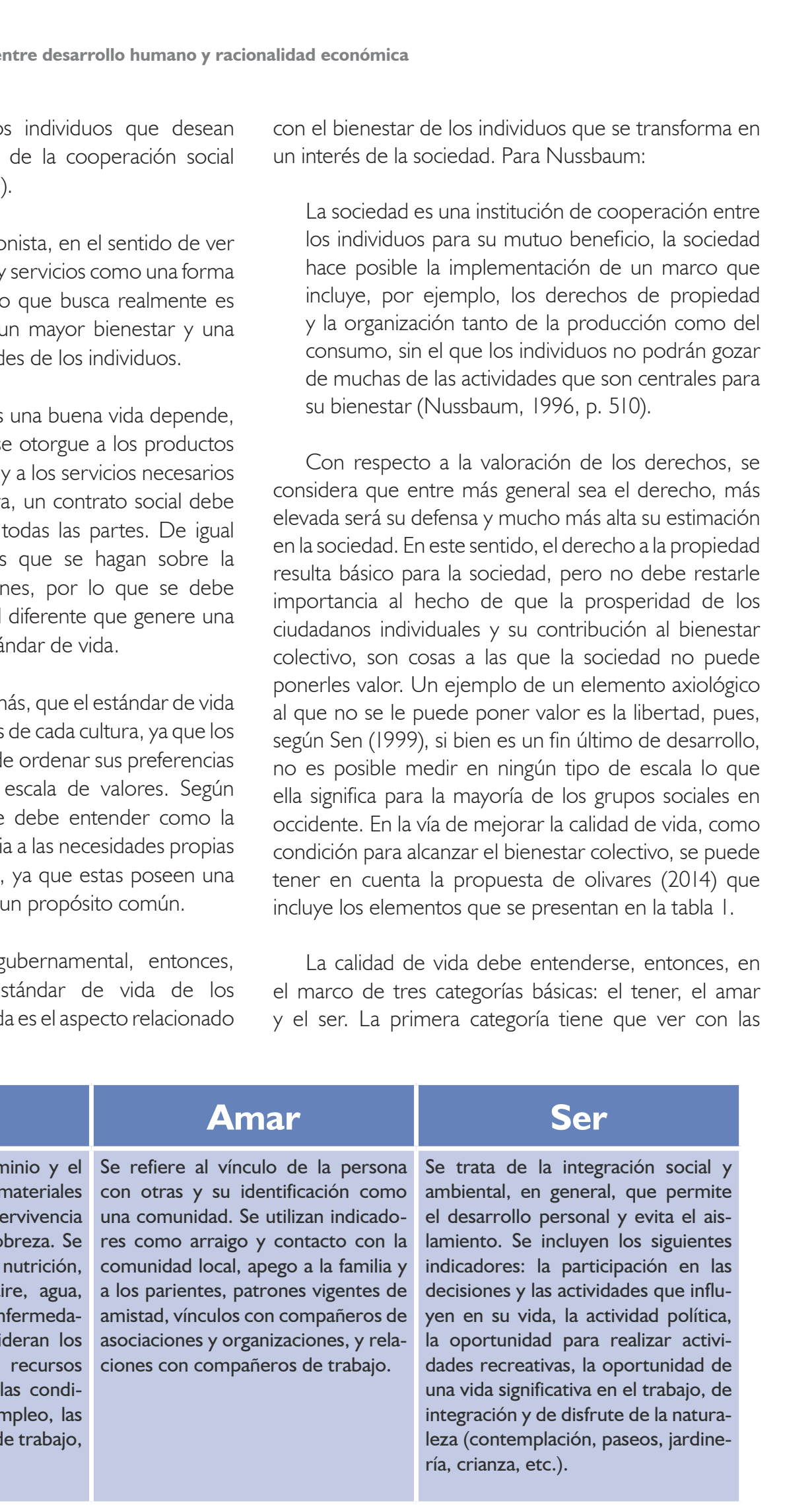


llamadas condiciones materiales que garantizan la vida; la segunda, con el mantenimiento de un vínculo o de una identificación con la comunidad a la que se pertenece y, la tercera, con la integración social, el estar en armonía con la naturaleza y los aspectos políticos, recreativos, espirituales y trascendentales. Si la calidad de vida reconoce e incluye como características básicas estas categorías, se podrá hablar de que es práctica y trascendente, y generará un mejor bienestar individual y colectivo, asegurando un mayor campo de acción para la elección individual, lo cual dependerá de la educación recibida.

Otra forma de categorizar las necesidades básicas de los seres humanos es la propuesta por Gough y Doyal (1994). Los autores definen como necesidades intermedias aquellas que están relacionadas con la autonomía y la supervivencia, reconociendo la existencia de dos clases de necesidades básicas humanas categorizadas en dos grupos o dimensiones, las cuales se deben garantizar para que el ser humano pueda actuar en sociedad de una manera efectiva; esta división corresponde a la salud física - que tendrá que ver con las necesidades básicas de supervivencia-y las necesidades básicas de autonomía.

De acuerdo con la división propuesta por estos autores, dentro de las necesidades humanas intermedias o categorizadas como de supervivencia, se encuentran, como elementos fundamentales, la alimentación nutritiva y el agua potable y el alojamiento adecuado, que se resumen en una vivienda digna: un espacio físico que se encuentre exento de riesgos y con

\begin{tabular}{|}
$\begin{array}{c}\text { Necesidades humanas de supervivencia } \\
\text { (intermedias) }\end{array}$ \\
\hline Alimentos nutritivos y agua potable \\
Alojamiento adecuado \\
\hline Medio físico desprovisto de riesgos \\
\hline Atención sanitaria adecuada
\end{tabular}

una adecuada atención sanitaria. Estos elementos serían básicos, en términos de los mínimos exigidos, para la supervivencia humana. En una segunda categoría se encuentran la seguridad a la infancia, las relaciones con personas que se consideran significativas desde el punto de vista del vínculo generado, la seguridad física, que tienen que ver con la salud; la seguridad económica, la cual se relaciona con la garantía de los ingresos mínimos para la supervivencia, y una enseñanza adecuada, no solo en el sentido de que los contenidos sean propicios para articularse al sistema productivo, sino en la generación de una formación holística que privilegie el tópico trascendental del ser humano, garantizando la posibilidad de adquirir autonomía para poder tomar las decisiones adecuadas.

\section{El enfoque DE CAPACIDADES}

Sen (1999) define capacidad como la manera en que las personas transforman su ingreso en aquello que consideran necesario para llevar la vida que quieren. El desarrollo se basa en la libertad, justamente porque esta permite a los individuos aumentar las capacidades que les permitan vivir de la forma en que quieran, lo cual, según su perspectiva, es lo que significa en esencia alcanzar un mayor desarrollo. Es obvio que el nivel de escogencia de aquello que se considera como lo más valioso para vivir, aumentará con el nivel educativo que la persona alcance en su proceso y con un mayor acceso a la formación. Según Edo (2002), el uso que podemos dar a un determinado nivel de ingreso depende de varias circunstancias. Sen (1999) identifica
Necesidades de autonomía (intermedias)

Seguridad de la infancia

Relaciones primarias significativas

Seguridad física

Seguridad económica

Enseñanza adecuada

Tabla 2. Clasificación de las necesidades básicas humanas

Nota: Adaptado de Teoría de las necesidades humanas, I. Gough y L. Doyal, 1994, Icaria. 
al menos cinco fuentes de diferencias entre la forma en que las personas establecen elecciones con respecto a sus ingresos sobre la base de sus necesidades básicas y su nivel educativo:

- Heterogeneidad personal: Las personas tienen características físicas diferentes de acuerdo con la edad, el sexo, la propensión a enfermarse, etc. La compensación necesaria para contrarrestar las desventajas varía, y algunas desventajas no pueden ser compensadas con mayores ingresos, sino con el acceso a la educación.

- Diversidad relacionada con el medio ambiente: El clima, por ejemplo, puede influir en lo que obtiene una persona de un determinado nivel de ingresos.

- Diferencias de clima social: Se trata de las condiciones sociales, incluidos los sistemas de enseñanza, el nivel de delincuencia, la violencia, la epidemiología y la contaminación.

- Diferencias entre las perspectivas relacionales: Los bienes derivados de las exigencias de las costumbres de un lugar pueden variar de una región a otra.

- Distribución dentro de las familias: De las reglas de distribución que se utilicen dentro de la familia pueden depender los logros y las dificultades económicas de sus miembros.

\section{Según Sen:}

Si el fin es centrar la atención en las oportunidades reales del individuo para alcanzar sus objetivos habría que tener en cuenta no sólo los bienes primarios que poseen las personas sino también las características personales relevantes que determinan la conversión de los bienes primarios en la capacidad de la persona para alcanzar sus fines (Sen, 1999, p. 117).

Esta forma de redefinir el desarrollo en términos de capacidades afecta directamente la manera en la que se concibe la pobreza, que no puede ser definida solamente a través del índice de la renta sino mediante las estrategias para poder eliminarla sistemáticamente. La educación, entonces, se constituye como mecanismo para vencer la pobreza y potenciar las capacidades. El enfoque de capacidades expuesto por Nussbaum (1996) -como se ha visto- se define a partir del concepto de capacidad, entendido como un conjunto de combinaciones y alternativas que permiten el funcionamiento del ser humano y que le resulta factible de alcanzar a una persona. Viene a ser una especie de libertad sustantiva, que busca combinaciones alternativas de funcionamientos (es decir, las combinaciones deben ser lógicas, en el sentido de que el individuo debe percibir, desde sus propios dispositivos cognitivos, sociales y trascendentales, que le generan un mayor bienestar), lo que incluye, como es obvio, las libertades o las oportunidades creadas por las facultades personales y el entorno político, social y económico. Para la autora, fomentar las capacidades es promover áreas de libertad, y es la potenciación de las capacidades la que garantiza la existencia de un espacio para el ejercicio de la libertad humana.

Este enfoque proporciona elementos para la construcción de una teoría de la justicia social y de los derechos, que se corresponde con el respeto a la facultad de autodeterminación que tienen las personas. El enfoque de capacidades, por tanto, se ocupa también de la injusticia y la desigualdad arraigadas, que se deben, especialmente, a la discriminación o la marginación, pero, además, a una pésima distribución del ingreso: el aumento de la brecha entre los percentiles que concentran la riqueza y de aquellos que tienen baja renta.

De igual manera, este enfoque concibe a cada persona, dentro del contexto del desarrollo humano, como un fin en sí misma; y, debido a las oportunidades disponibles para cada ser humano, se centra en la posibilidad de elegir, o sea la libertad, pues considera que las sociedades deben proporcionar el conjunto de las oportunidades que las personas pueden llevar o no llevar a la práctica, siendo ellas quienes elijan. El Estado y las políticas públicas tienen como responsabilidad de mejorar la calidad de vida para el conjunto de la sociedad, tarea en la que las capacidades tienen una influencia importante. Nussbaum (1996) habla, además, de capacidades sustanciales o combinadas, que son el conjunto de las oportunidades de las que dispone una persona para elegir y para actuar en su situación particular dentro de un contexto político, social y económico; en este concepto son relevantes las características de una persona:

- Rasgos de personalidad

- Capacidades intelectuales y emocionales

- Estado de salud y forma física

- Aprendizaje interiorizado

- Habilidades de percepción y movimiento 
Solo se puede ver el desarrollo de capacidades combinadas cuando la sociedad procura el desarrollo de las capacidades internas de las personas; estas tienen que ver realmente con los rasgos o aptitudes entrenadas en interacción con el entorno social, político, económico y familiar. Dentro de esta concepción de desarrollo humano se afirma que la posibilidad de que las facultades de las personas puedan desplegarse depende de la superación de un umbral de capacidad combinada y de la posibilidad para el sujeto de elegir y actuar.

El funcionamiento, que, como se había visto, consiste en la realización activa de una o más capacidades, tiene una doble implicación: se constituye en la materialización, por medio de los actos, en nuevas formas de combinación de alternativas, y la adquisición de nuevas capacidades. En este sentido, el funcionamiento de las capacidades combinadas tiene que ver con la idea de dignidad humana y se relaciona estrechamente con la noción de conación activa, que se entiende como aquella parte del sujeto relacionada con sus anhelos, esfuerzos y deseos. La conación activa se emparenta con lo que el sujeto espera de su vida.

Los Estados tienen un margen de acción para desarrollar las capacidades de los ciudadanos, siempre y cuando estas estén en concordancia con sus tradiciones y su cultura. En este sentido, la seguridad de la capacidad hace referencia a que el Estado no solo debe proporcionar una capacidad a las personas, debe además facilitarla de tal manera que pueda contar con ella posteriormente. El funcionamiento fértil, al que se refiere Nussbaum (1996), es aquel que busca favorecer otras capacidades, y la desventaja corrosiva, constituye el grupo de las privaciones que tienen efectos graves en varios aspectos de la vida. En la política pública se debería tratar de profundizar acerca de las capacidades, los funcionamientos fértiles y las desventajas corrosivas, permitiendo que se constituya un criterio estándar que realice comparaciones entre cuestiones de igualdad, considerando que esta es un valor político importante. De esta manera, las capacidades ejercen un papel en diferentes niveles y procesos que, siguiendo a Nussbaum (1996), se presentan en la tabla 3:

En contraste, Sen (1996), se centra en un uso comparativo que destaca en su estructura algunas capacidades como, por ejemplo, la salud, la educación, la participación política y la no discriminación de raza, religión y género. Esto constituye una idea de justicia básica que se aproxima a la propuesta de Nussbaum (1996), en su concepción de capacidades centrales. El enfoque de capacidades, que introduce la teoría de la filosofía moral en la economía del desarrollo, no solo se fundamenta en las preferencias subjetivas, sino que, al contrario, se ubica en contra de los enfoques que se basan en las preferencias de las personas, ya que considera que estas no son fiables en cuanto a sus efectos políticos.

Dentro de este enfoque también se presenta otro tipo de teorías filosóficas que abordan el concepto de desarrollo humano, como por ejemplo las bienestaristas, que están basadas en las preferencias que tienen los
Principios políticos

Ser utilizada como una fuente de principios políticos durante un periodo de diseño constitucional o como una fuente para la interpretación de esa constitución.
Derechos fundamentales

Guiar la interpretación judicial de derechos fundamentales, aunque dentro de unos límites fijados por el texto constitucional.

Tabla 3. Niveles de ejecución de las capacidades

Nota: Autor (2015) a partir de los planteamientos teóricos de Nussbaum y Sen, en La calidad de vida, 1996, Fondo de Cultura Económica. 
sujetos. Se incorporan, por ejemplo elementos de tipo moral, que son claves para este enfoque:

(...) La idea de la igualdad humana de todas las personas, la idea de la importancia fundamental de la razón práctica como capacidad, la idea de que las personas no deberían tener la prerrogativa de suprimir los derechos fundamentales de otras (Nussbaum, 1996, p. 105).

Finalmente, la propuesta de Nussbaum (1996) resulta más profunda que la de las capacidades que plantea Sen (1996), ya que su intención es lograr que las personas reflexionen en torno a lo que es una sociedad mínimamente justa. Si bien este enfoque de las capacidades, que al ser potenciadas permiten una mejora en la calidad de vida y una mayor libertad en la elección, se relaciona con una categorización necesaria de dichas capacidades, lo que busca, en realidad, es apoyarse en el hecho de que el homo œconomicus es considerado por naturaleza como un agente racional.

\section{RACIONALIDAD ECONÓMICA}

Según Streb (1998), se puede distinguir entre una racionalidad en sentido limitado (maximización de beneficio) y una racionalidad en sentido amplio (optimización). El autor considera que la racionalidad es un principio a nivel individual que no necesariamente debe coincidir con la racionalidad colectiva. Las fallas de esta racionalidad se pueden relacionar con problemas de racionalidad acotada y con inconsistencias en nuestras preferencias; sin embargo, sin distinción frente a la racionalidad de la que se esté hablando, se debe reconocer que esta debe contener algunos elementos o criterios básicos. Al respecto Tversky y Kahneman (198I) indican: "Sobre la definición de racionalidad se ha discutido mucho, pero hay un consenso general en que las elecciones racionales deben satisfacer algunos requisitos elementales como la consistencia y la coherencia" (p. 453 -traducción del autor-).

Estos criterios entran en contradicción con elementos subjetivos que motivan a los individuos a tomar decisiones que no necesariamente se relacionan con racionalidades no acotadas. Bunge (Citado por Streb, 1998), llama al principio de racionalidad "extremismo economicista" y lo compara con la codicia. Parece existir una confusión entre el deseo de maximizar el lucro con el de racionalidad; en realidad, lo que supone la racionalidad individual es que las personas saben o aprenden debido a la llamada "racionalidad expost", ya que al evaluar sus propios intereses y aprender de ellos mismos -en un ejercicio de metacognición- se logra analizar el proceso de la práctica racional.

Incluso, economistas que defienden en extremo la libertad de elegir reconocen limitaciones en el principio de racionalidad. Friedman (1963), por ejemplo, sostiene que las decisiones se exigen de individuos responsables, por lo cual los enfermos mentales y los menores de edad estarían limitados para ejercer la libertad, y agrega que es difícil encontrar el límite para determinar que un ser humano sea realmente libre para elegir. Se plantean las siguientes preguntas: ipuede resolver parte de la idea de racionalidad en economía, el hecho de observar el grado de maduración del individuo según su ciclo vital?, ide qué forma influyen los procesos psicológicos en las decisiones económicas? Una persona racional sabe qué prefiere, cuáles son sus gustos; esto es lo que le permite tomar una decisión. El individuo racional debe ser, por lo tanto, un individuo inteligente y emocionalmente maduro (Streb, 1998).

Los economistas han considerado, casi siempre como un axioma implícito de la ciencia económica, la existencia de una única racionalidad capaz de explicar todo el comportamiento humano, incluidos los instintos, los sentimientos de odio, la filantropía, la solidaridad, etc. De hecho, el concepto de homo œconomicus ha sido utilizado siempre como equivalente -o por lo menos muy cercano- al de racionalidad económica. Un homo œconomicus consistiría en un conjunto de supuestos en los que se basarían los criterios que llevan a la decisión económica de los individuos. Se podría argumentar que han existido, y pueden existir, diversos homo œconomicus; pero cada uno de ellos quedaba definido por su racionalidad, por su forma de ordenar preferencias $y$, por tanto, tendría una única y diferente racionalidad. Hayek, por ejemplo, fue el primero en cuestionar la consideración del comportamiento instintivo como irracional y sugirió la existencia de una especie de racionalidad intermedia o semirracionalidad que estaría situada, según sus propias palabras, entre el instinto y la razón. En su obra La fatal arrogancia: errores del socialismo (1990) se hace énfasis en un supuesto carácter antisocial de la moral existente en la sociedad tribal y una especie de moral antitribal de la sociedad capitalista:

Si pretendiéramos aplicar las rígidas pautas de conducta propias del microcosmos (es decir, del orden que caracteriza a la convivencia en la pequeña banda o mesnada, e incluso en la propia unidad familiar) al macrocosmos (es decir, al orden propio de la sociedad civilizada en toda su complejidad y extensión) -como tan reiteradamente nos 
recomiendan nuestras profundas tendenciaspondríamos en peligro a ese segundo tipo de orden. Y si, a la inversa, pretendiéramos aplicar la normativa propia del orden extenso a esas agrupaciones más reducidas, acabaríamos con la misma cohesión que las aglutina (Hayek, 1990, p. 50 -traducción del autor-).

Es imposible hablar de una única y general racionalidad, y la influencia en ella dependerá no solo de los contextos sociales, sino además de las condiciones emocionales y psicológicas de quien toma la decisión.

En esta línea argumental se debe ir mucho más lejos de lo que plantea Hayek, reconociendo la existencia de diversas y variadas racionalidades, cada una de ellas asociada a una forma o sistema económico, conviviendo de manera simultánea y, como resulta obvio, generando situaciones conflictivas. La exploración de las limitaciones de la racionalidad todavía no está plenamente integrada en la teoría económica. Simon (1979), por ejemplo, contrasta la racionalidad perfecta (no acotada), que supone que no existen limitaciones computacionales o analíticas para encontrar la solución óptima a un problema, con la idea de racionalidad acotada. El concepto de racionalidad acotada resume lo que John M. Keynes (1973) entendió como su contribución fundamental a la economía y que marca su principal separación con la teoría económica anterior. Para este autor es imposible conocer el futuro; la base poco firme de nuestras expectativas individuales sobre el futuro, las flimsy foundations de puntos de vista fundados en extrapolaciones del presente y en las opiniones existentes en la sociedad, lleva a que estas expectativas estén sujetas a cambios bruscos y violentos.

Se pueden acercar ambos enfoques agregando limitaciones informativas y computacionales a las restricciones que enfrentan decisores perfectamente racionales. Keynes enfatiza en que los seres humanos, a lo sumo, pueden aspirar a encontrar una solución satisfactoria para la mayoría de los problemas, ya que son demasiado complejos para poder ser resueltos a la perfección.

Algo todavía menos explorado que la racionalidad acotada es el problema de conocer bien los propios gustos, que forma parte de la madurez emocional que discute Akerlof (1991). Se sabe, a veces, que si no se limitan las opciones, se pueden tomar decisiones que después habrá que lamentar. El ejemplo clásico es el de Ulises: sabía este que no iba a poder resistir el canto de las sirenas por lo que pidió ser atado por sus marineros: una contradicción entre los gustos y la racionalidad. El individuo, además de ser inconsistente con sus propios gustos, puede ser también influenciable en el momento en que toma decisiones dentro de un grupo; poco a poco puede ir cambiando su personalidad y terminar haciendo cosas que nunca hubiera sido capaz de hacer por su propia cuenta, incluso que pueden desagradarlo mucho, fenómeno que se conocen como la obediencia indebida.

Si se estudiaran -reconociendo las bases etológicas subyacentes-aspectos relativos a la toma de decisiones, se podría argumentar que existe una especie de racionalidad natural, asociada a un sistema de economía natural, en la que las leyes naturales y físicas son las que influyen de manera efectiva en las decisiones, seleccionando individuos de especies biológicas que toman determinadas decisiones. Existe una especie de racionalidad instintiva, asociada de nuevo a un sistema de economía instintiva, en la que el individuo toma de manera inconsciente sus decisiones en función de informaciones que poco a poco va adquiriendo en su ciclo vital.

En la sociedad contemporánea existe una racionalidad, que se podría llamar tradicional, asociada sobre todo al sistema de economía tradicional, en la que las decisiones las toman los individuos según las estimaciones de coste y valor basadas en criterios que han construido con el tiempo. Existe, además, una racionalidad política, asociada al sistema de la economía política, en la que las decisiones generalmente se basan en esquemas de coste y valor y sustentadas en cálculos articulados expresables en términos de derechos. Podría hablarse, incluso, de una racionalidad financiera, que estaría asociada a un sistema de economía financiera, en el que las decisiones las toman los individuos según algunas estimaciones de coste y valor basadas en cálculos formales que generalmente se expresan en términos monetarios. Estas dos últimas formas de racionalidad son mucho más pragmáticas, pues responden a la lógica y al desarrollo de las fuerzas productivas del capitalismo tardío, mientras que la primera se relaciona con las creencias y costumbres construidas culturalmente.

Las diversas racionalidades se apoyan unas en otras; parece existir entre ellas una relación triangular y transitiva. La racionalidad financiera supondría el reconocimiento y la necesidad de los derechos; es decir, de la racionalidad política. A su vez, la racionalidad política debería apoyarse en los principios de autoridad y tradición, sin los cuales los derechos no existirían, mientras que la aceptación de estos principios como criterios de valoración y adopción de decisiones -lo que se ha denominado como "racionalidad tradicional", 
estaría fundamentada en el instinto y, posteriormente, en el hábito, o por lo menos tendría una imponderable influencia de este.

A pesar de que estas racionalidades estén interrelacionadas, convivirían de manera simultánea, pero de forma independiente en todos los individuos. Ante un conjunto de alternativas, es posible que el instinto proponga al sujeto una ordenación racional (transitiva) determinada; la tradición, otra ordenación diferente; el sistema de derechos otra más y así sucesivamente. Es posible que las diversas racionalidades planteen ordenaciones diferentes y que estas entren en conflicto, sobre todo porque utilizan diversos sistemas para las mediciones de costes y estimaciones de valores; es por eso que, frecuentemente, los seres humanos tienen dificultades a la hora de tomar una decisión. En este sentido, la decisión debe fundamentarse en criterios de bienestar y utilidad.

\section{CONCLUSIONES}

Estas ideas iníciales permiten abordar puntos de encuentro entre los planteamientos de desarrollo humano y racionalidad económica. Pareciese que la relación entre ambos conceptos fuera de interdependencia; es decir, el desarrollo humano sería un elemento básico para cualquier tipo de racionalidad o la racionalidad implicaría como elemento básico no solo la maximización de beneficios individuales sino colectivos. En este sentido, el punto de coincidencia relativo al concepto de capacidad-racionalidaddesarrollo, puede desplegarse aún más en el marco de la libertad, en la medida en que dichas capacidades se potencien desde la educación y en una racionalidad que propicie las acciones colectivas. Más que un conjunto de afirmaciones, el artículo genera nuevos campos de discusión; los temas básicos para seguir investigando tienen que ver con la relación entre los tipos de racionalidad y las decisiones de los sujetos sobre la base de sus procesos psicológicos superiores (percepción, motivación, memoria, etc.), la importancia de la subjetividad y su influencia en la racionalidad y el desarrollo, la forma en que se potencian dichas capacidades y cómo contribuirán estas al avance del desarrollo humano en nuestras sociedades.

Si bien los aportes de la teoría del bienestar y el utilitarismo resultan relevantes dentro de la teoría económica en la explicación de la toma de decisiones de los sujetos entre aquellas alternativas que les resulten más viables, se puede encontrar, también, un vasto campo de estudio que puede involucrar los aspectos subjetivos que determinan, en muchos casos, esas decisiones y los teoremas que definen clásicamente la elección de los agentes considerados desde la modernidad como racionales.

De tal forma, el científico social, llámese psicólogo o economista, encuentra de manera habitual comportamientos que no pueden explicarse desde un único sistema de racionalidad. Los economistas han intentado, a menudo, conciliar esas contradicciones y explicar una racionalidad acudiendo a otras racionalidades. Se ha querido convencer a propios y ajenos de que había una única racionalidad: la que estudiaba y trataba de comprender la ciencia económica. Lo que intenta este texto, principalmente, es ahondar en las diferentes formas de racionalidad y comprender las distintas situaciones en las que una racionalidad prevalece sobre las otras. Sin embargo, se reconoce que en situaciones de riesgo y en estudios de aversión al riesgo, la teoría que mejor explica el comportamiento en términos de elecciones internas es el modelo de utilidad esperada.

Los experimentos realizados muestran un nuevo campo de análisis y de relación entre la ciencia económica y los procesos psicológicos superiores que generan, por lo tanto, un nuevo campo de investigación que permitirá la ampliación del concepto de racionalidad económica, en función del bienestar, la utilidad y, de forma mucho más profunda, del el desarrollo \& 


\section{Referencias bibliográficas}

Akerlof, G. A. (Mayo, 1991). Procrastination and obedience (Papers and Proceedings of the Hundred and Third Annual Meeting of the American Economic Association). The American Economic Review, 8I (2), I-I9.

Argibay, M., Celorio, G. y Celorio, J. J. (Agosto, 1997). Educación para el desarrollo: El Espacio olvidado de la cooperación. Cuadernos de Trabajo Hegoa, 19 (monográfico).

Brandts, J. (2007). La economía experimental y la economía del comportamiento. Barcelona: Instituto de Análisis Económico -CISC.

Calderón, F. (Julio, 2003). El desarrollo humano como búsqueda de una sociedad mejor. PNUD (dirección), ponencia presentada en el Taller Alcanzando entre todos Excelencia e Impacto, de los Informes Nacionales de Desarrollo Humano -INDH, realizado en Santa Cruz de la Sierra, Bolivia.

Cruz, J. (2003). Daniel Kahneman: Un nuevo Premio Nobel de Economía para la psicología. Revista Latinoamericana de Psicología, 35(I), II9-I2I.

Edo, M. (2002). Amartya Sen y el desarrollo como libertad: La viabilidad de una alternativa a las estrategias de promoción del desarrollo (Tesis de grado). Licenciatura en Estudios Internacionales, Universidad Torcuato di Tella, Buenos Aires, Argentina.

Friedman, M. (1963). Capitalism and Freedom. Chicago: University of Chicago Press.

Gough, I. y Doyal L. (1994). Teoría de las necesidades humanas. Barcelona: Icaria.

González, J. (Dir.). (2008). Bogotá una apuesta por Colombia: Informe de desarrollo humano. Bogotá, D. C.: PNUD.

Hayek, F. (1990). The Fatal Conceit: The Errors of Socialism. Londres: Routledge.

Hernández, T. (Primavera, 2004). Cultura, diversidad y desarrollo humano. Quorum, 17, 59-63.

Keynes, J. (1974). Teoría general de la ocupación el interés y el dinero. México, D. F: Fondo de Cultura Económica.

Max, M. (1993). Desarrollo a escala humana: Conceptos, aplicaciones y reflexiones. Barcelona: Icaria.

Max, M. (1993). Economía descalza: Señales desde el mundo visible. Montevideo: Nordan.

Olivares, J. (2014). Desarrollo sustentable: Unidad IV, Calidad de Vida y Desarrollo Sustentable (material de apoyo del curso Desarrollo Sustentable, Tema 4). Recuperado de http://desarrollosustentable2.bligoo.com.mx/media/users//8/945493/files/210287/material4ds.pdf

Posas, M. (Julio, 2008). Exclusión social, inclusión social y desarrollo humano. PNUD, Revista Latinoamericana de Desarrollo Humano, 46.

Rawls, J. (2006). Teoría de la justicia. México, D. F.: Fondo de Cultura Económica.

Nussbaum, M y Sen, A. (1996). La calidad de vida. México, D. F.: Fondo de Cultura Económica.

Smith, V. (Abril, 1962). An Experimental Study of Competitive Market Behavior. Journal of Political Behavior, 70(2), III-I37.

Sen, A. (1997). Sobre la desigualdad económica. Madrid: Folio.

Sen, A. (1999). Desarrollo como libertad. Buenos Aires: Planeta.

Simon, H. (Septiembre, 1979). Rational decision making in business organizations. American Economic Review, 69(4), 493-5I3.

Sorman, G. (1999). Los grandes pensadores de nuestro tiempo. Bogotá, D. C.: Planeta.

Streb, J. (1998). El significado de la racionalidad económica (Documento de trabajo Universidad de CEMA).

Varian, P. (1998). Microeconomía intermedia: Un enfoque Actual (4ª Ed.). Barcelona: Antoni Bosch.

Tversky, A. y Kahneman, D. (Septiembre, 1974). Judgement Under Uncertainty: Heuristic and Biases. Science (Nez Series), I85(4I57), II24-II3I.

Tversky, A. y Kahneman, D. (Marzo, 1979). Prospect Theory: An Analysis of Decision under Risk. Econometrica, 47(2), $263-292$.

Tversky, A. y Kahenman, D. (Enero, 198I). The Framing of Decisions and the Psychology of Choice. Science (New Series), 21 I (448I), 453-458. 